\title{
Will There Be a Vaccine to Prevent HCV Infection?
}

\author{
Jonathan R. Honegger, MD ${ }^{1,2}$ Yan Zhou, $\mathrm{PhD}^{1} \quad$ Christopher M. Walker, PhD ${ }^{1,2}$
}

${ }^{1}$ The Center for Vaccines and Immunity, Nationwide Children's Hospital, Columbus, Ohio

2 Department of Pediatrics, College of Medicine, The Ohio State University, Columbus, Ohio

\begin{abstract}
Address for correspondence Chris Walker, PhD, Nationwide Children's Hospital, 700 Children's Drive, WA4011, Columbus, OH 43205

(e-mail: christopher.walker@nationwidechildrens.org).
\end{abstract}

Semin Liver Dis 2014;34:79-88.

\begin{abstract}
Keywords

- hepatitis C virus

- vaccine

- direct acting antiviral

- persistent infection

Prevention of hepatitis $\mathrm{C}$ virus ( $\mathrm{HCV}$ ) infection by vaccination has been a priority since discovery of the virus and the need has not diminished over the past 25 years. Infection rates are increasing in developed countries because of intravenous drug use. Reducing transmission will be difficult without a vaccine to prevent persistence of primary infections, and also secondary infections that may occur after cure of chronic hepatitis $C$ with increasingly effective direct-acting antiviral (DAA) regimens. Vaccine need is also acute in resource poor countries where most new infections occur and DAAs may be unaffordable. Spontaneous resolution of HCV infection confers durable protection, but mechanisms of immunity remain obscure and contested in the context of vaccine design. A vaccine must elicit a CD4+ helper T cell response that does not fail during acute infection. The need for neutralizing antibodies versus cytotoxic CD8+ T cells is unsettled and reflected in the design of two very different vaccines evaluated in humans for safety and immunogenicity. Here we review the status of vaccine development and the scientific and practical challenges that must be met if the burden of liver disease caused by HCV is to be reduced or eliminated.
\end{abstract}

\section{The Need for a Vaccine 25 Years after the Discovery of HCV}

The hepatitis C virus (HCV) was identified in 1988 as the major cause of transfusion and community acquired non- $A$ non-B hepatitis. ${ }^{1}$ The enormous scope of the global public health problem was rapidly defined, and it was determined that approximately three-quarters of all infections persist for life and substantially increase the risk of progressive liver diseases. Twenty-five years later, in 2013, the first all-oral antiviral regimen to replace type I interferon for treatment of some HCV infections was approved in the United States, a historic advance against this virus that has infected over $2 \%$ of the world's population. ${ }^{2}$ A rich pipeline of direct-acting antiviral (DAA) drugs in phase II and phase III trials promises to yield additional regimens with markedly improved efficacy, safety, and convenience compared with traditional inter-

Issue Theme New Hepatitis C Therapies; Guest Editor, Jean-Michel Pawlotsky, MD, $\mathrm{PhD}$ feron-based therapies. ${ }^{3}$ There is hope that widespread use of these potent agents will fundamentally alter the HCV epidemic, stemming the surge of HCV-related cirrhosis and hepatocellular carcinoma that is expected to peak in the coming decades, ${ }^{4,5}$ and preventing new HCV infections by shrinking the pool of infectious persons. ${ }^{6}$ With development of DAAs active against all HCV genotypes, even global eradication of HCV is a theoretical possibility. ${ }^{6,7}$

Thus, the question arises, is a vaccine to prevent HCV infection still needed and, if so, who would it protect? Answers to these important questions depend on the feasibility of achieving widespread DAA treatment uptake and the ultimate goals of HCV control efforts. Potential roles for an HCV vaccine range from protection of select populations with identifiable risk of infection to implementation of universal vaccination programs. Two populations that could be reasonably targeted for vaccination include health care workers with
Copyright $\odot 2014$ by Thieme Medical Publishers, Inc., 333 Seventh Avenue, New York, NY 10001, USA. Tel: +1(212) 584-4662.
DOI http://dx.doi.org/ 10.1055/s-0034-1371081. ISSN 0272-8087. 
occupational risk of blood exposure and persons who are cured by DAA therapy but remain at risk of re-exposure to the virus through injection drug use (IDU). Because antiviral cures may not restore or impart protective immunity, an effective vaccine administered after costly DAA-mediated cure could provide relatively inexpensive insurance against a second persistent HCV infection. This approach to vaccination could conceivably tip the balance in favor of treating subjects who carry this risk.

A broad vaccine strategy to disrupt HCV transmission would likely be necessary to realize the more ambitious goal of a major reduction in the global HCV burden. Injection drug use is the primary route of transmission in industrialized nations where DAA therapy will be most widely deployed. ${ }^{5}$ Despite years of prevention efforts, IDU associated acute HCV diagnoses are increasing in certain populations such as young adults in the United States. ${ }^{8,9}$ Sobering reports from numerous state health departments and correctional facilities document a doubling of HCV diagnoses among adolescents and young adults over the past decade due to escalating epidemics of IDU and needle sharing. ${ }^{10-12} \mathrm{Al}-$ though modeling studies of urban IDU cohorts suggest that aggressive HCV screening and DAA treatment of active users could substantially reduce HCV prevalence and transmission, ${ }^{13,14}$ an increasing proportion of IDU associated HCV infections occur in rural parts of the United States where fewer public health resources exist for intensive case finding and treatment. ${ }^{8,10,12}$ DAA regimens will be priced close to the cost of interferon-based regimens that they will replace, likely exceeding $\$ 100,000$ in the United States. ${ }^{5}$ These prices are considered cost effective relative to the expense of HCV complications, ${ }^{6}$ but could render widespread uptake of DAA treatment unaffordable even in high-income nations. An effective vaccine provided to uninfected IDUs could over a decade or more substantially reduce incidence of new HCV infections in IDU cohorts. ${ }^{15}$ Given the inherent difficulties of identifying active IDU for treatment, comprehensive strategies incorporating both DAA treatment with prophylactic and posttreatment vaccination may have the best chances of substantially reducing the HCV burden in IDU cohorts in the developed world.

It is notable that the HAV and HBV vaccines were initially deployed selectively to prevent infection in those with identifiable risk, but their full impact on global liver disease was not realized until implementation of universal vaccination in regions of the world where the viruses cause greatest harm. Most of the 185 million HCV seropositive individuals live in developing countries ${ }^{2}$ where iatrogenic transmissions via contaminated injections and transfusions remain major portals of infection. ${ }^{16,17}$ The silent nature of the HCV pandemic and anticipated costs of DAA therapies present even more formidable obstacles to the "treatment as prevention" approach to HCV infection control in these settings. It is estimated that fewer than one in six are aware of their infection, and massive HCV screening programs will be required to significantly scale-up treatment to the point where new infections would be prevented. ${ }^{2,5}$ This will almost certainly be problematic in settings where the health care infrastructure lacks the capacity to provide safe infection control practices. DAA costs might be sharply discounted for lower-income countries, as for HIV antiretrovirals, if similar public pressure, licensing policies, and private/public funding are applied. ${ }^{6}$ Nevertheless, even if pricing were reduced 1,000-fold in low-income nations, costs of large-scale HCV screening and DAA treatment could far exceed those of a program to prevent infection by vaccination. Specific targeting of an $\mathrm{HCV}$ vaccine to those at greatest risk in developed countries (IDU) or developing countries (iatrogenic and IDU) will be difficult and so development of a vaccine that is compatible with universal distribution will be important if preventing transmission is the goal.

Here we review what is known about correlates of protective immunity against HCV, current approaches and status of HCV vaccine development, and the scientific challenges that must still be overcome for success.

\section{Is It Possible to Vaccinate against HCV?}

In the modern era of vaccine development there has been remarkable progress in preventing infection with hepatotropic viruses. The current hepatitis A (HAV) and B virus (HBV) vaccines, licensed in the United States in 1986 and 1995 , respectively, have substantially reduced the burden of liver disease over the past two decades as universal immunization programs have become more common in many developed and developing countries. ${ }^{18}$ A vaccine that prevents hepatitis E virus (HEV) infection has potential to further reduce liver disease if deployed in regions of the world where endemic and epidemic spread is common. ${ }^{18}$ The HAV, HBV, and HEV vaccines share three important characteristics. First, they are comprised of recombinant subunit proteins (HBV surface protein and HEV capsid) or whole inactivated virus (HAV) and are straightforward to produce from a technical and cost perspective. Second, these immunogens elicit durable antibody responses that are strongly correlated with apparent protection from infection, defined as sterilizing immunity. Third, the HAV, HBV, and HEV vaccines are monovalent, but effectively prevent infection in all regions of the world regardless of local diversity in viral genotype or serotype.

HCV presents a very different and much more difficult target for vaccination. At least seven HCV genotypes that vary in nucleotide sequence by $\sim 30 \%$ have been described globally. ${ }^{19}$ Considerable variation is localized to domains of the HCV envelope glycoproteins targeted by neutralizing antibodies. The HCV genome is also highly mutable because of an error prone RNA polymerase that facilitates evasion of host immune responses, perhaps including those generated by vaccination..$^{20,21}$ Most importantly, unlike the other hepatitis viruses, resolution of acute HCV infection does not necessarily confer sterilizing immunity upon re-exposure to the virus. Early experiments documented virus replication in chimpanzees that were rechallenged after complete control of an earlier HCV infection. ${ }^{22,23}$ In some cases, sequential infections were caused by the identical strain of HCV. ${ }^{23,24}$ 
The absence of sterilizing immunity was initially interpreted as worrisome for development of a protective HCV vaccine. With the development of more robust assays to quantify HCV replication, it became apparent that although immune chimpanzees were susceptible to reinfection, the duration and magnitude of viremia was often substantially reduced, even when a different genotype of the virus was used for challenge. ${ }^{25-30}$ Attenuated HCV replication has also been described in humans who successfully controlled an earlier primary infection. ${ }^{31,32}$ Most importantly, secondary infec- tions in chimpanzees ${ }^{25-30}$ and humans ${ }^{31-33}$ are much more likely to resolve than primary infections. In humans, it is estimated that $80 \%$ of secondary infections resolve spontaneously compared with $30 \%$ of primary infections (-Fig. 1A). ${ }^{31,32}$

Symptoms of acute primary and secondary hepatitis $C$ are typically mild or even inapparent so vaccination to generate sterilizing immunity is not an imperative. There is no longterm or irreversible damage to the liver when the infection resolves spontaneously. Moreover, HCV replication does not

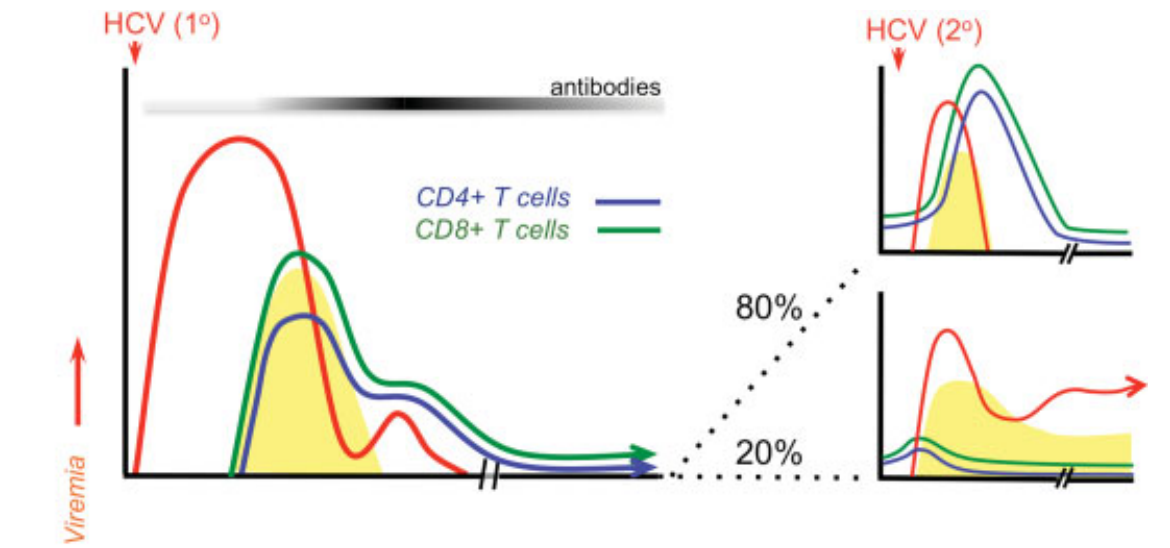

A

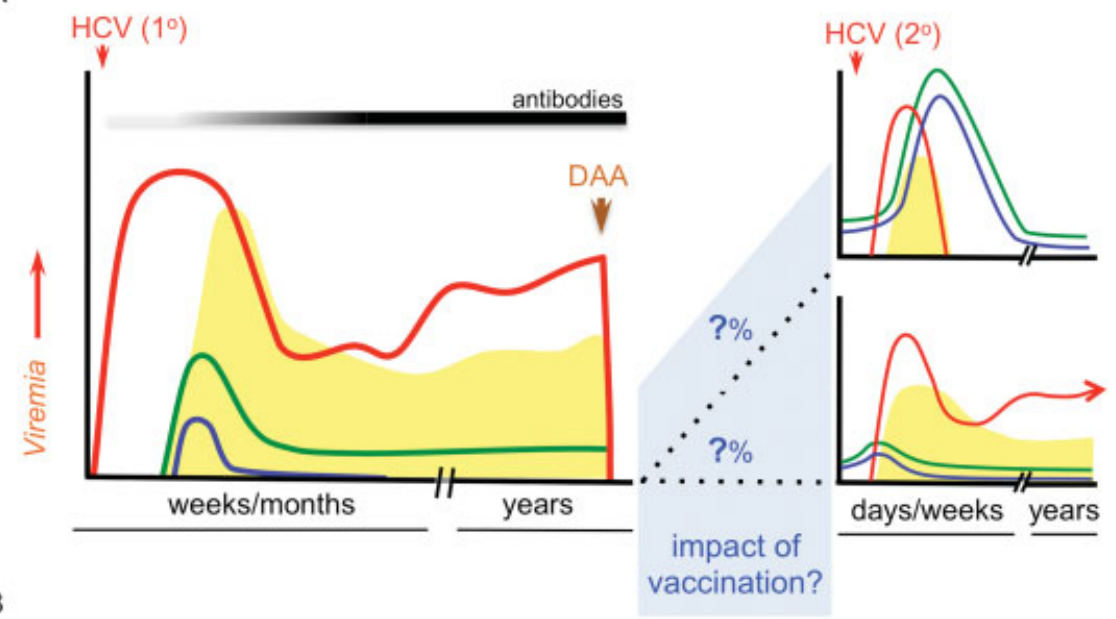

Fig. 1 (A) Immunity during and after resolution of primary hepatitis C virus (HCV) infection. Left panel. Approximately $30 \%$ of acute primary HCV infections resolve spontaneously. Viremia typically peaks at $\sim 8$ to 12 weeks after infection and drops precipitously with expansion of circulating CD4+ and CD8+ T cells. Transaminases (yellow shaded area) usually peak coincident with the onset of HCV-specific T-cell immunity. Importantly, CD4+ and CD8+ T-cell immunity is sustained until well after apparent resolution of infection and long-lived memory populations persist in blood and liver for years to decades. Seroconversion to HCV proteins occurs as acute infection is controlled and neutralization of contemporaneous viruses is common. Anti-HCV antibody titers can decline after resolution of infection. Right panels. Viremia is observed after secondary HCV infection, but $\sim 80 \%$ resolve spontaneously and only $20 \%$ persist. Secondary infections can resolve within days, viremia and liver transaminases are substantially reduced compared with primary infection, and control of HCV replication is associated with rapid recall of T-cell immunity and possibly neutralizing antibodies. One goal of HCV vaccination is to generate in unexposed humans the long-lived immunity induced by spontaneous control of infection. (B) Persistent HCV infection and the uncertain influence of direct-acting antiviral (DAA) therapy on immunity and secondary infection. Left panel. Approximately $70 \%$ of primary HCV infections persist. CD4+ T cell responses fail before the virus is cleared. CD8+ T cells become exhausted or select for escape mutations in class I epitopes. Seroconversion to HCV proteins occurs after several weeks to months of infection. Neutralization of contemporaneous viruses is uncommon. The status of CD4+ T cells, CD8+ T cells and neutralizing antibodies after DAA-mediated cure is unknown. Right panels. The fraction of secondary infections that resolve or persist after DAA cure is unknown (blue shaded area). The rate of resolution will be considerably less than the $80 \%$ observed after spontaneous clearance of a first infection, if HCV-specific adaptive immune responses are permanently impaired or fail to generate memory as expected. A second goal of preventive vaccination is to repair any long-lasting damage to HCV-specific humoral and/or cellular immune responses after DAA cure so that the rate of persistence upon reinfection is reduced (blue shaded area). Vaccination after cure will be a critical adjunct to DAA therapy if future research demonstrates that CD4+ T helper cells do not respond to secondary infection and the repertoire of CD8+ T cells against intact, previously escaped, and/or new epitopes unique to the reinfecting virus is restricted. 
relapse once the acute infection resolves spontaneously, and only rarely in patients who clear the virus after type I interferon and ribavirin treatment, ${ }^{34,35}$ so there is limited risk of a long-lived cellular reservoir of virus genomes to reinitiate infection if immunity weakens. Collectively, these observations provide a compelling argument that it is possible to vaccinate against $\mathrm{HCV}$, but with a very different objective when compared with the other major hepatitis viruses. For HCV, the most realistic goal of vaccination is not to induce sterilizing immunity, but instead to skew acute $\mathrm{HCV}$ infection toward resolution and away from persistence.

\section{Defining Protective Immunity against HCV Infection}

A detailed understanding of immune mechanisms that contribute to rapid control of an acute HCV infection would aid development of a vaccine to prevent persistence. However, correlates of protective immunity are still not completely defined after 25 years of study in humans and chimpanzees. There is still considerable uncertainty about the relative contribution of humoral versus cellular immune responses to resolution of primary and secondary HCV infection.

\section{Humoral Immunity}

A key unresolved question is whether antibodies facilitate resolution of acute infections. Evidence supporting this possibility is mixed. HCV infections do resolve in some humans with primary hypogammaglobulinemia ${ }^{36}$ and in some chimpanzees that do not develop antibodies against the HCV envelope glycoproteins. It is also notable that passive transfer of hepatitis $\mathrm{C}$ immunoglobulin ${ }^{37}$ or a neutralizing anti-E2 monoclonal antibody ${ }^{38}$ to chimpanzees immediately after virus challenge delayed the onset of viremia and acute hepatitis, but had no obvious impact on the course of infection. Collectively, these observations argue against an absolute requirement for antibodies in control of an established acute infection.

Humans do generate an antibody response to envelope glycoproteins E1 and E2 regardless of whether the infection ultimately persists or resolves. Early broad neutralizing activity could conceivably reduce the risk of HCV persistence in the context of a broader adaptive immune response. Functional antibody responses in patients with resolved and chronic infections have been compared using HCV pseudoparticles (designated HCVpp) that display HCV envelope glycoproteins on a VSV or retroviral capsid. Early studies using HCVpp bearing envelope glycoproteins of standard HCV laboratory strains found no association between infection outcome and the titer of serum neutralizing antibodies. A more accurate picture of neutralization was obtained when antibodies and envelope glycoproteins used to construct HCVpp were derived from the same patient or relevant donor. In one of the first studies of this design, Lavillette and colleagues measured neutralizing antibody responses against a virus transmitted by hemodialysis. ${ }^{39}$ Among the 17 patients studied, those with the lowest levels of viremia had the highest serum neutralizing antibody titers against the trans- mitted (donor) virus. A subsequent study of pregnant women accidentally infected with HCV after treatment with a common lot of contaminated immunoglobulin documented higher rates of virus clearance in those with acute phase antibodies that blocked HCVpp entry into cultured hepatocytes. ${ }^{40}$ Other studies documented that neutralization is usually weak in primary infections that persist and is associated with emergence of immune escape variants. ${ }^{41,42}$ Recent case reports have also documented an association between the onset of neutralizing antibody responses and spontaneous control of HCV replication anywhere from a few weeks to more than a year after infection. ${ }^{43}$ Whether immune humans develop neutralizing antibodies after reinfection with the virus has not been widely studied and so their role in rapid termination of a second infection remains uncertain. In one study, 10 of 12 secondary infections in humans resolved spontaneously. ${ }^{32}$ A broad neutralizing antibody response was observed in a subset of these cases, including the two that resulted in persistent replication of the virus. ${ }^{32}$

It is important to emphasize that most studies of serum antibody neutralization were retrospective in nature and so T-cell immunity was not measured contemporaneously in the same subjects. It is somewhat difficult to disentangle the relative contribution of humoral versus cellular immunity to resolution of infection in the few studies where both responses were measured. ${ }^{32,43}$

\section{Cellular Immunity}

Evidence from human and chimpanzee studies supports a role for HCV-specific CD4+ helper and CD8+ cytotoxic T cells in control of primary and secondary HCV infections. A temporal kinetic association between the appearance of HCV-specific T cells in blood and an initial sharp drop in viremia, usually at $\sim 8$ to 12 weeks after primary infection, provided the first evidence that cellular immunity might be important to control of acute hepatitis C (-Fig. 1A). ${ }^{44,45}$ Follow-up studies revealed that T-cell responses are often qualitatively different in resolving and persisting infections. The frequency of HCV-specific T cells in blood is generally greater in subjects who clear the infection when quantified by direct visualization with MHC tetramers or production of antiviral cytokines. ${ }^{44,45}$ Successful primary responses also tend to be broader, targeting more $\mathrm{HCV}$ proteins or epitopes. ${ }^{44,45}$ More recent studies in chimpanzees suggest that phenotypic differences might distinguish sustained T-cell responses from those that fail before virus is cleared. CD8+ T cells from animals that controlled HCV replication were more likely to express CD127, the interleukin-7 receptor $\alpha$ subunit that is marker of memory precursors, during the early phase of infection when they were still viremic. ${ }^{46} \mathrm{CD} 8+\mathrm{T}$ cells from animals that clear infection are also more likely to have a phenotype associated with proliferation (Ki67 positive/Bcl-2 low) and activation (HLA-DR positive). ${ }^{47}$ Programmed cell death 1 (PD1 ), which is expressed on CD8 + T cells in chronic hepatitis $C$ and may contribute to the exhausted phenotype, was not predictive of $\mathrm{HCV}$ persistence at the early stages of infection in these chimpanzees. ${ }^{46}$ 
It is important to emphasize that chronic hepatitis $C$ sometimes develops despite early robust expansion of CD4 + and CD8 + T cells that target multiple class II and I epitopes. ${ }^{43,44}$ These individuals can exhibit a substantial drop in viremia and prolonged period of partial control of virus replication before persistence is established ( $\mathbf{- F i g}$. 1B). To date the best predictor of a persistent course is premature failure of the CD4+ T helper cell response. ${ }^{43,44,48}$ Loss of CD4+ $\mathrm{T}$ cells that produce antiviral cytokines and/or proliferate in response to HCV antigen stimulation has been associated with resurgent virus replication. ${ }^{49}$ Once lost, the helper response is rarely re-established and persistent infection ensues. The absence of CD4 + T cell help is thought to impair CD8 + T cells immunity, resulting in functional exhaustion and selection of HCV variants that carry escape mutations in class I epitopes. $^{43,44}$

HCV-specific T-cell memory generated after resolution of primary infection is thought to contribute to rapid control of a second infection in humans and chimpanzees. In representative studies, reinfection of chimpanzees with $\mathrm{HCV} \sim 7$ years after resolution of a first infection resulted in substantial acceleration of the T-cell response. ${ }^{28,29}$ Virus-specific memory $\mathrm{T}$ cells reached peak frequency within 7 to 10 days compared with 10 to 12 weeks after the first infection. HCV replication was controlled within 14 days and viremia was several orders of magnitude lower than in the first infection. ${ }^{28,29}$ Antibodymediated depletion of CD8 $+\mathrm{T}$ cells ${ }^{29}$ immediately before a third infection of these animals prolonged viremia while depletion of CD4+ T cells ${ }^{28}$ resulted in persistence instead of rapid resolution. These observations provided direct evidence that memory $\mathrm{T}$ cells are an important component of protection in immune individuals who are re-exposed to the virus. It is important to emphasize that some secondary infections in humans and chimpanzees ${ }^{30,50}$ do persist despite memory Tcell immunity and so protection is not absolute. In one remarkable example, an immune chimpanzee developed a persistent infection after multiple sequential challenges with different strains and genotypes of HCV. ${ }^{50}$

\section{Models for Preventive Vaccination}

Studies of immunity and HCV replication during the acute and chronic phases of infection suggest models for preventive vaccination of humans. A successful vaccine would ideally reduce the magnitude and duration of primary viremia and skew infection outcome strongly toward resolution (-Fig. 1A). Induction of protective immunity may be relatively straightforward for HCV-naïve vaccinees. There is considerable uncertainty around the status of immunity in those who are cured using interferon-free DAA regimens and remain at risk for reinfection (- Fig. 1B). The permanency of immune system dysfunction after cure has not been explored. It is possible that some CD8 + T cells, especially those that are less exhausted because cognate class I epitopes escaped early in infection, ${ }^{51-53}$ would respond upon re-exposure to virus. Whether CD8 $+\mathrm{T}$ cells could respond to epitopes that remained intact in the first infection, or recognize a new repertoire of class I epitopes, is much more difficult to predict. Protection will depend on whether the CD4+ T cell compart- ment is restored after DAA cure. It is unlikely that effective memory $\mathrm{CD} 4+\mathrm{T}$ cell populations would emerge under this circumstance (-Fig. 1B). A new CD4 $+\mathrm{T}$ cell response that resembles the one generated after primary infection may be the best case scenario. It is likely that the level of protection in those cured of persistent infection would be no better, and perhaps worse, than the $30 \%$ rate of spontaneous resolution observed in HCV naïve subjects. Preventive vaccination after DAA-mediated cure may therefore be required to provide a consistently high level of protection from reinfection for those at risk (-Fig. 1B).

Finally, studies of HCV infection and immunity have not yet provided clear correlates of protective immunity. The possibility that an early humoral response that broadly neutralizes contemporaneous viruses can contribute to termination of infection cannot be excluded. Strong cellular immunity is a hallmark of infections that resolve, but only when sustained well past the point of apparent clearance of the virus. Whether a vaccine must prime antibodies or CD8+ $\mathrm{T}$ cells is a matter of considerable debate and reflected in divergent approaches to vaccination reviewed below. There is a consensus that strong CD4 $+\mathrm{T}$ cell immunity that does not fail late in the course of acute hepatitis $C$ is essential. An absence of hypotheses to explain premature loss of HCVspecific CD4 $+\mathrm{T}$ cells, or how it might be prevented by vaccination, remains a very significant blind spot for HCV vaccine development. There are as yet no reliable surrogate markers to predict whether a CD4+ T cell response generated by infection or vaccination is destined to succeed or fail.

\section{Current Status of HCV Vaccines}

Traditional approaches involving attenuation or inactivation of whole virus have received little consideration for vaccination against HCV. Generation of a live-attenuated HCV vaccine with no ability to persist, subvert immunity, or cause progressive liver disease is for now a conceptual and practical impossibility. A classical whole-killed vaccine could not be considered until the relatively recent development of cell culture systems that facilitate replication of HCV. One genotype 2 virus produced in the HCV cell culture system was inactivated and used to immunize mice. ${ }^{54}$ Sera from these animals inhibited infectivity of genotype 1 and 2 viruses in a hepatocyte cell culture model and the genotype 2 virus in immunodeficient mice with humanized livers. ${ }^{54}$

The discovery of HCV coincided with a revolution in recombinant DNA technology to produce virus proteins and vectors and knowledge of how antigens are processed for $\mathrm{CD} 8+\mathrm{T}$ cell priming. These advances provided new options for the design of vaccines to elicit humoral and cellular immune responses.

\section{Vaccines to Generate Neutralizing Antibodies}

The subunit HBV surface antigen, licensed as a vaccine 2 years before the discovery of $\mathrm{HCV}$, was the first to be produced using recombinant DNA technology. This advance provided the initial impetus for development of recombinant subunit E1 and E2 envelope glycoproteins as a vaccine by Chiron 
Corporation (now Novartis, Basel, Switzerland), with the goal of generating protective neutralizing antibodies. Heterodimeric vaccines containing HCV genotype 1 E1 and E2 glycoproteins, when combined with an adjuvant, elicited strong humoral immune responses in nonhuman primates. ${ }^{55,56}$ More detailed analysis of antibodies raised with a recombinant E1/E2 heterodimer in an oil-water emulsion adjuvant revealed broad neutralizing function against multiple HCV genotypes as assessed in the HCVpp cell culture assay. ${ }^{57}$ In a series of chimpanzee challenge studies, adjuvanted E1/E2 vaccines sometimes provided sterilizing immunity against genotype 1 challenge viruses. ${ }^{55}$ Breakthrough infections were also observed. ${ }^{55,56}$ Although some of these infections persisted, a comparison of controls and vaccinated animals from multiple studies revealed that resolution was much more common in the latter group. ${ }^{55,58}$ Antibodies alone are almost certainly not sufficient to terminate HCV infection once it is established, and so an important contribution of CD4+ T cells primed by the vaccines can't be excluded. The possibility that the vaccine-primed E1/E2-specific CD4+ T cells are resistant to inactivation during acute HCV infection, and facilitate priming of antibodies and CD8 $+\mathrm{T}$ cells that contribute to clearance, merits further study.

The recombinant E1/E2 heterodimer combined with an oil-water adjuvant developed by Chiron/Novartis has been assessed for immunogenicity in human volunteers not at risk for infection. Strong CD4+ T cell responses were detected in lymphoproliferation assays and serum antibodies inhibited entry of genotype $1 \mathrm{HCVpp}$ into hepatocytes. ${ }^{59,60}$ A very recent reanalysis of serum from a subset of patients documented the potential for very broad pan-genotypic neutralization of HCV after vaccination with E1/E2 derived from a single genotype 1 isolate of the virus. ${ }^{61}$ No further progress in clinical development of this vaccine has been reported and so its fate is uncertain.

Other approaches to elicit anti-HCV antibodies have been evaluated in animal models. Expression of HCV envelope glycoproteins from plasmid DNA, ${ }^{62}$ and recombinant virus vectors have been used to induce antibodies in chimpanzees. Several of the virus vectors incorporated HCV E1 and/or E2 and nonstructural genes to induce CD8 $+\mathrm{T}$ cells and are reviewed below. Finally, prime-boost strategies long favored for vaccination against HIV may also have utility for HCV. One recent study documented that recombinant viral vectors and synthetic virus like particle (VLP) triggers incorporating HCV E1 and E2 elicited broadly neutralizing antibodies in macaques. $^{63}$ Design of advanced synthetic immunogens may also be facilitated by crystallographic analysis of the interaction between broadly neutralizing monoclonal antibodies and the HCV glycoproteins. ${ }^{64}$

\section{Vaccines to Generate HCV-Specific T Cells}

At the time HCV was discovered, key steps were taken toward understanding how antigens are processed for priming of cytotoxic CD8 + T lymphocytes. Townsend, McMichael, and colleagues documented in 1986 that CD8 + T cells recognize short viral peptides presented on the cell surface by class I MHC molecules. ${ }^{65}$ Subsequent studies rapidly defined the pathway for processing antigenic peptides from viral proteins produced in the cytoplasm. ${ }^{66}$ These advances sparked tremendous innovation in vaccine technologies to prime $\mathrm{CD} 8+\mathrm{T}$ cells. The newly discovered HCV provided a potentially relevant target for proof of concept studies. A wide array of replicating and nonreplicating virus vectors, plasmid DNA, pooled synthetic class I epitopes, virus-like particles (VLP), and adjuvants that facilitate antigen delivery to the cytosol for class I antigen processing have been adapted for use as HCV vaccines. Genetic vaccines, including plasmid DNA or recombinant virus vectors, have been most commonly used. They typically express nonstructural proteins like NS3, NS4, and/or NS5 because they are the dominant target of CD8+ T cells and well-conserved across HCV genotypes when compared with the envelope glycoproteins. ${ }^{67}$ VLP are the exception; they are comprised of the HCV envelope glycoproteins and core protein. ${ }^{68}$ All of these concept vaccines primed CD8+ T cells in rodents, a relatively low hurdle for immunogenicity. A very small number of vaccines were shown to elicit robust CD8 $+\mathrm{T}$ cell immunity in the blood and/or liver of macaques and baboons. ${ }^{63,69-74}$ Fewer have been assessed for immunogenicity and protection of chimpanzees from HCV challenge. They included a VLP comprised of the HCV E1, E2, and core proteins, ${ }^{68}$ recombinant nonstructural proteins formulated with the iscomatrix adjuvant, and genetic vaccines that encoded nonstructural proteins. ${ }^{20,75-77}$ As noted above, some genetic vaccines also encoded envelope glycoproteins that induced antibodies, but none provided sterilizing immunity. ${ }^{75-77}$ Collectively these vaccines reduced primary viremia after challenge with $\mathrm{HCV},{ }^{20,68,75-77}$ some by several orders of magnitude as summarized in a meta-analysis of all chimpanzee vaccine studies. ${ }^{58}$ Suppression of acute-phase virus replication was associated with recall of vaccine-primed T cells. ${ }^{58}$ The outcome of infection in vaccinated chimpanzees was, however, highly variable in these studies..$^{20,58,68,77,78}$ In general, individual vaccine studies included too few animals, and approaches to analysis of cellular immunity were too varied, to draw conclusions about the vectors, antigens, and nature of $\mathrm{T}$-cell responses that provided protection. That vaccine design might have a profound influence on infection outcome even when the same nonstructural proteins are used to prime $\mathrm{T}$ cells is illustrated by two studies. In the first example, acute-phase HCV replication was substantially suppressed in five chimpanzees vaccinated with recombinant NS3, NS4, and NS5 proteins formulated with the iscomatrix adjuvant when compared with the mock-vaccinated controls. ${ }^{55}$ However, all vaccinated animals, but none of the controls developed a chronic infection. In the second example, priming and boosting of chimpanzees with recombinant adenoviruses and plasmid DNA encoding the NS3, NS4, and NS55 proteins provided a very different result. ${ }^{76}$ Sharp suppression of acute-phase viremia was also observed in all five animals that received this vaccine. However, the infection was ultimately controlled in four individuals after several weeks of very low level, intermittent viremia. ${ }^{76} \mathrm{~A}$ follow-up study revealed that after challenge, $C D 8+T$ cells induced by vaccination with the recombinant adenovirus and plasmid DNA had sustained expression of CD127, lower levels of PD-1, 
and enhanced effector functions when compared with primary $\mathrm{T}$ cells from the mock-vaccinated controls that developed persistent infections. ${ }^{79}$ This result obtained with the iscomatrix-based vaccine raised the troubling possibility that some antigen and adjuvant combinations have the potential to unpredictably enhance persistent infection. The reason for very different infection outcomes with these vaccines despite suppression of acute phase viremia is not known. The frequency of circulating HCV-specific $\mathrm{T}$ cells that produced interferon- $\gamma$ after vaccination and virus challenge was determined in all vaccine studies, but this measure alone appears to be insufficient to predict infection outcome. ${ }^{58}$ More detailed phenotypic and functional analyses will be needed to gain insight into factors that determine if a vaccine will reduce or possibly even increase the rate of persistent infection in humans.

Phase I safety and immunogenicity trials of two vaccines designed to prevent infection by eliciting T-cell immunity have been completed. Immunogenicity of an HCV core protein formulated with the iscomatrix adjuvant was determined in human volunteers not at risk for HCV infection. T cell responses were detected in only a subset of vaccinees. Phase I testing of a prime-boost regimen with the recombinant adenovirus vectors encoding NS3, NS4, and NS5 developed by Okairos (now Glaxo Smith Kline) was also undertaken in humans, supported by the promising outcome of the chimpanzee studies. ${ }^{80}$ Vaccination resulted in CD4+ and CD8 $+\mathrm{T}$ cell immunity with attributes generally associated with control of virus infection. ${ }^{80}$ The T cells displayed multiple effector functions. Memory CD8 + T cells that expressed CD127 but not PD-1 were sustained in circulation. ${ }^{80}$ Importantly, epitopes conserved in HCV genotype 1 and 3 viruses were recognized, indicating the potential for cross-genotypic protection. A version of this vaccine involving a prime with recombinant adenovirus vector and boosting with modified vaccinia virus Ankara is now being evaluated in a staged phase I/II study in Baltimore and San Francisco (see ClinicalTrials.gov NCT01436357). The goal of this study is to prevent $\mathrm{HCV}$ persistence in $\mathrm{HCV}$-naïve IDU populations at high risk for infection.

\section{Will There Be a Vaccine to Prevent HCV Infection?}

Uncertainty about correlates of protective immunity is illustrated by very different approaches to the design of two vaccines that have been evaluated in humans. The Chiron/ Novartis vaccine comprised of E1 and E2 envelope glycoproteins in an oil-water emulsion adjuvant was designed to elicit neutralizing antibodies and helper CD4 $+\mathrm{T}$ cells required to generate a humoral immune response. Although immunogenicity data appeared promising, no plan for an efficacy trial in the foreseeable future is apparent. Alternative vaccines to assess role of antibodies and T-helper cells in protection from $\mathrm{HCV}$ infection and persistence are not on the horizon. The second vaccine developed by Okairos/GSK will test the hypothesis that a T-cell response primed with nonstructural $\mathrm{HCV}$ proteins is sufficient to reduce the incidence of chronic hepatitis $C$ by preventing persistence of the virus. It will also provide critical proof that large vaccine efficacy trials can be conducted in IDU, a largely marginalized population where basic needs like stable housing are often unmet and the risk of incarceration and even death is increased. ${ }^{81,82}$ Because of these unique challenges, it is estimated that the phase I/II trial will be $\sim 4$ years in duration. Whether this vaccine prevents persistence will not be known until early 2016 (ClinicalTrials. gov NCT01436357). Exactly how efficacious a vaccine like the one developed by Okairos/GlaxoSmithKline must be to have a material impact on liver disease in rural and urban IDU populations has not been widely studied. Modeling suggests that a vaccine targeted to IDU in San Francisco that is even 50\% efficacious would substantially reduce the incidence of chronic liver disease over a period of years or decades, but with the assumption of high vaccine uptake among those at risk of infection. ${ }^{15}$ Predictive modeling of how a combined program of vaccination and DAA treatment to reduce the virus donor pool would interrupt HCV transmission in rural and urban IDU populations is needed.

Progress toward a preventive HCV vaccine could be considered slow when judged by the limited number of candidates that have entered clinical testing. It is important to weigh progress against the considerable scientific and practical challenges that must be overcome to develop an HCV vaccine. As noted above, the timeframe from initiation to completion of HCV vaccine efficacy trials is measured in years and there is a paucity of at risk human cohorts and institutional infrastructure for this purpose. ${ }^{83}$ At the same time, animal models to validate vaccine concepts before human testing are limiting. New regulations in the United States define a highly restrictive set of conditions for use of chimpanzees in biomedical research. ${ }^{84} \mathrm{~A}$ variety of new infection models involving rodents and even lower nonhuman primates are under development and have been reviewed elsewhere. ${ }^{85}$ The pace of progress is rapid, but as yet no model recapitulates key features of HCV infection and immunity in humans. Advancing new vaccine concepts to phase I, and particularly phase II, human trials may be difficult without evidence that infection outcome is improved and not worsened in animals. Perhaps more importantly, iterative improvements to vaccine design may be required if protection in humans is less than optimal in initial efficacy trials. An empirical approach to vaccine development that involves cycles of evaluation in human subjects and improvements to increase efficacy will almost certainly require refinement of clinical trial design to shorten duration and/or a more tractable animal model with the same fidelity to human HCV infection and immunity provided by the chimpanzee. Vaccine trials may well provide the best insight into correlates of protective immunity, but will require phenotypic and functional analyses of adaptive immune responses in human vaccinees that are technically challenging, more similar to current vaccine development programs for HIV than past successful efforts for the other hepatitis viruses. It is likely that the imperative to develop HCV vaccines, and increase the pace of progress, will become more apparent as the cost of reinfection after DAA therapy is better defined in developed 
countries. Ideally, the outcome of this process will be a preventive, pan-genotypic HCV vaccine that is similar to other hepatitis virus vaccines in cost and ease of production for use in developed countries, but especially in developing regions of the world where most new HCV infections occur and DAA therapies may well be unaffordable.

\section{Acknowledgments}

This study was funded by Public Health Service grants R37 AI47367 to CMW and R01 AI096882 02 to CMW and JRH.

\section{References}

1 Choo QL, Kuo G, Weiner AJ, Overby LR, Bradley DW, Houghton M. Isolation of a cDNA clone derived from a blood-borne non- $A$, nonB viral hepatitis genome. Science 1989;244(4902):359-362

2 Mohd Hanafiah K, Groeger J, Flaxman AD, Wiersma ST. Global epidemiology of hepatitis $C$ virus infection: new estimates of agespecific antibody to HCV seroprevalence. Hepatology 2013;57(4): 1333-1342

3 Scheel TK, Rice CM. Understanding the hepatitis C virus life cycle paves the way for highly effective therapies. Nat Med 2013;19(7): 837-849

4 Davis GL, Alter MJ, El-Serag H, Poynard T, Jennings LW. Aging of hepatitis $\mathrm{C}$ virus (HCV)-infected persons in the United States: a multiple cohort model of HCV prevalence and disease progression. Gastroenterology 2010;138(2):513-521, e1-e6

5 Thomas DL. Global control of hepatitis C: where challenge meets opportunity. Nat Med 2013;19(7):850-858

6 Hagan LM, Wolpe PR, Schinazi RF. Treatment as prevention and cure towards global eradication of hepatitis $C$ virus. Trends Microbiol 2013;21(12):625-633

7 Torti C, Focà A, Carosi G. Towards HCV extinction with modern HCV treatment? “Yes we can!”. BMC Infect Dis 2012;12(Suppl 2):S1

8 Klevens RM, Hu DJ, Jiles R, Holmberg SD. Evolving epidemiology of hepatitis C virus in the United States. Clin Infect Dis 2012;55 (Suppl 1):S3-S9

9 Lin RH, Hwang YW, Yang BC, Lin CS. TNF receptor-2-triggered apoptosis is associated with the down-regulation of Bcl-xL on activated T cells and can be prevented by CD28 costimulation. J Immunol 1997;158(2):598-603

10 Centers for Disease Control and Prevention (CDC). Hepatitis C virus infection among adolescents and young adults:Massachusetts, 2002-2009. MMWR Morb Mortal Wkly Rep 2011;60(17): 537-541

11 McNamara BC, Losikoff PT, Huguenin L, Macalino GE, Rich JD, Gregory SH. Increasing Hepatitis C Prevalence and Associated Risk Behaviors among Incarcerated Young Adults. J Urban Health 2013

12 Centers for Disease Control and Prevention (CDC). Notes from the field : hepatitis $C$ virus infections among young adults-rural Wisconsin, 2010. MMWR Morb Mortal Wkly Rep 2012;61(19):358

13 Rolls DA, Sacks-Davis R, Jenkinson R, et al. Hepatitis C transmission and treatment in contact networks of people who inject drugs. PLoS ONE 2013;8(11):e78286

14 Martin NK, Vickerman P, Grebely J, et al. Hepatitis C virus treatment for prevention among people who inject drugs: Modeling treatment scale-up in the age of direct-acting antivirals. Hepatology 2013;58(5):1598-1609

15 Hahn JA, Wylie D, Dill J, et al. Potential impact of vaccination on the hepatitis C virus epidemic in injection drug users. Epidemics 2009; 1(1):47-57

16 Hauri AM, Armstrong GL, Hutin YJ. The global burden of disease attributable to contaminated injections given in health care settings. Int J STD AIDS 2004;15(1):7-16
17 Miller FD, Abu-Raddad LJ. Evidence of intense ongoing endemic transmission of hepatitis C virus in Egypt. Proc Natl Acad Sci U S A 2010;107(33):14757-14762

18 Lavanchy D. Viral hepatitis: global goals for vaccination. J Clin Virol 2012;55(4):296-302

19 Smith DB, Bukh J, Kuiken C, et al. Expanded classification of hepatitis C Virus into 7 genotypes and 67 Subtypes: Updated criteria and assignment web resource. Hepatology 2014;59(1): 318-327

20 Zubkova I, Choi YH, Chang E, et al. T-cell vaccines that elicit effective immune responses against $\mathrm{HCV}$ in chimpanzees may create greater immune pressure for viral mutation. Vaccine 2009; 27(19):2594-2602

21 Puig M, Mihalik K, Tilton JC, et al. CD4+ immune escape and subsequent $\mathrm{T}$-cell failure following chimpanzee immunization against hepatitis C virus. Hepatology 2006;44(3):736-745

22 Prince AM. Immunity in hepatitis C virus infection. Vox Sang 1994; 67(Suppl 3):227-228

23 Farci P, Alter HJ, Govindarajan S, et al. Lack of protective immunity against reinfection with hepatitis C virus. Science 1992; 258(5079):135-140

24 Prince AM, Brotman B, Huima T, Pascual D, Jaffery M, Inchauspé G. Immunity in hepatitis C infection. J Infect Dis 1992;165(3): 438-443

25 Major ME, Mihalik K, Puig M, et al. Previously infected and recovered chimpanzees exhibit rapid responses that control hepatitis C virus replication upon rechallenge. J Virol 2002;76(13): 6586-6595

26 Bassett SE, Guerra B, Brasky K, et al. Protective immune response to hepatitis $C$ virus in chimpanzees rechallenged following clearance of primary infection. Hepatology 2001;33(6):1479-1487

27 Lanford RE, Guerra B, Chavez D, et al. Cross-genotype immunity to hepatitis C virus. J Virol 2004;78(3):1575-1581

28 Grakoui A, Shoukry NH, Woollard DJ, et al. HCV persistence and immune evasion in the absence of memory $\mathrm{T}$ cell help. Science 2003;302(5645):659-662

29 Shoukry NH, Grakoui A, Houghton M, et al. Memory CD8+ T cells are required for protection from persistent hepatitis $\mathrm{C}$ virus infection. J Exp Med 2003;197(12):1645-1655

30 Prince AM, Brotman B, Lee DH, et al. Protection against chronic hepatitis $C$ virus infection after rechallenge with homologous, but not heterologous, genotypes in a chimpanzee model. J Infect Dis 2005;192(10):1701-1709

31 Grebely J, Prins M, Hellard M, et al; International Collaboration of Incident HIV and Hepatitis C in Injecting Cohorts (InC3). Hepatitis $C$ virus clearance, reinfection, and persistence, with insights from studies of injecting drug users: towards a vaccine. Lancet Infect Dis 2012;12(5):408-414

32 Osburn WO, Fisher BE, Dowd KA, et al. Spontaneous control of primary hepatitis $C$ virus infection and immunity against persistent reinfection. Gastroenterology 2010;138(1):315-324

33 Mehta SH, Cox A, Hoover DR, et al. Protection against persistence of hepatitis C. Lancet 2002;359(9316):1478-1483

34 Hara K, Rivera MM, Koh C, et al. Sequence analysis of hepatitis C virus from patients with relapse after a sustained virological response: relapse or reinfection? J Infect Dis 2014;209(1):38-45

35 Lee WM, Polson JE, Carney DS, Sahin B, Gale M Jr. Reemergence of hepatitis $C$ virus after 8.5 years in a patient with hypogammaglobulinemia: evidence for an occult viral reservoir. J Infect Dis 2005; 192(6):1088-1092

36 Semmo N, Lucas M, Krashias G, Lauer G, Chapel H, Klenerman P. Maintenance of HCV-specific T-cell responses in antibody-deficient patients a decade after early therapy. Blood 2006;107(11): 4570-4571

37 Krawczynski K, Alter MJ, Tankersley DL, et al. Effect of immune globulin on the prevention of experimental hepatitis $C$ virus infection. J Infect Dis 1996;173(4):822-828 
38 Morin TJ, Broering TJ, Leav BA, et al. Human monoclonal antibody HCV1 effectively prevents and treats HCV infection in chimpanzees. PLoS Pathog 2012;8(8):e1002895

39 Lavillette D, Morice Y, Germanidis G, et al. Human serum facilitates hepatitis $C$ virus infection, and neutralizing responses inversely correlate with viral replication kinetics at the acute phase of hepatitis C virus infection. J Virol 2005;79(10):6023-6034

40 Pestka JM, Zeisel MB, Bläser E, et al. Rapid induction of virusneutralizing antibodies and viral clearance in a single-source outbreak of hepatitis C. Proc Natl Acad Sci U S A 2007;104(14): 6025-6030

41 Dowd KA, Netski DM, Wang XH, Cox AL, Ray SC. Selection pressure from neutralizing antibodies drives sequence evolution during acute infection with hepatitis C virus. Gastroenterology 2009; 136(7):2377-2386

42 von Hahn T, Yoon JC, Alter H, et al. Hepatitis C virus continuously escapes from neutralizing antibody and T-cell responses during chronic infection in vivo. Gastroenterology 2007;132(2):667-678

43 Raghuraman S, Park H, Osburn WO, Winkelstein E, Edlin BR, Rehermann B. Spontaneous clearance of chronic hepatitis $C$ virus infection is associated with appearance of neutralizing antibodies and reversal of T-cell exhaustion. J Infect Dis 2012;205(5): 763-771

44 Rehermann B. Hepatitis $C$ virus versus innate and adaptive immune responses: a tale of coevolution and coexistence. J Clin Invest 2009;119(7):1745-1754

45 Walker CM. Adaptive immunity to the hepatitis $\mathrm{C}$ virus. Adv Virus Res 2010;78:43-86

46 Shin EC, Park SH, Nascimbeni M, et al. The frequency of CD127(+) hepatitis $\mathrm{C}$ virus (HCV)-specific $\mathrm{T}$ cells but not the expression of exhaustion markers predicts the outcome of acute HCV infection. J Virol 2013;87(8):4772-4777

47 Zubkova I, Duan H, Wells F, et al. Hepatitis C virus clearance correlates with HLA-DR expression on proliferating CD8+ T-cells in immune-primed chimpanzees. Hepatology 2013

48 Schulze Zur Wiesch J, Ciuffreda D, Lewis-Ximenez L, et al. Broadly directed virus-specific $\mathrm{CD} 4+\mathrm{T}$ cell responses are primed during acute hepatitis $C$ infection, but rapidly disappear from human blood with viral persistence. J Exp Med 2012;209(1):61-75

49 Gerlach JT, Diepolder HM, Jung MC, et al. Recurrence of hepatitis C virus after loss of virus-specific CD4(+) T-cell response in acute hepatitis C. Gastroenterology 1999;117(4):933-941

50 Bukh J, Thimme R, Meunier JC, et al. Previously infected chimpanzees are not consistently protected against reinfection or persistent infection after reexposure to the identical hepatitis $C$ virus strain. J Virol 2008;82(16):8183-8195

51 Rutebemberwa A, Ray SC, Astemborski J, et al. High-programmed death-1 levels on hepatitis $C$ virus-specific $T$ cells during acute infection are associated with viral persistence and require preservation of cognate antigen during chronic infection. J Immunol 2008;181(12):8215-8225

52 Bengsch B, Seigel B, Ruhl M, et al. Coexpression of PD-1, 2B4, CD160 and KLRG1 on exhausted HCV-specific CD8+ T cells is linked to antigen recognition and T cell differentiation. PLoS Pathog 2010; 6(6): 1000947

53 Kasprowicz V, Kang YH, Lucas M, et al. Hepatitis C virus (HCV) sequence variation induces an HCV-specific T-cell phenotype analogous to spontaneous resolution. J Virol 2010;84(3): 1656-1663

54 Akazawa D, Moriyama M, Yokokawa $\mathrm{H}$, et al. Neutralizing antibodies induced by cell culture-derived hepatitis $C$ virus protect against infection in mice. Gastroenterology 2013;145(2):447-455, e1-e4

55 Houghton M. Prospects for prophylactic and therapeutic vaccines against the hepatitis C viruses. Immunol Rev 2011;239(1):99-108

56 Puig M, Major ME, Mihalik K, Feinstone SM. Immunization of chimpanzees with an envelope protein-based vaccine enhances specific humoral and cellular immune responses that delay hepatitis C virus infection. Vaccine 2004;22(8):991-1000
57 Meunier JC, Gottwein JM, Houghton M, et al. Vaccine-induced cross-genotype reactive neutralizing antibodies against hepatitis C virus. J Infect Dis 2011;204(8):1186-1190

58 Dahari H, Feinstone SM, Major ME. Meta-analysis of hepatitis C virus vaccine efficacy in chimpanzees indicates an importance for structural proteins. Gastroenterology 2010;139(3):965-974

59 Frey SE, Houghton M, Coates S, et al. Safety and immunogenicity of HCV E1E2 vaccine adjuvanted with MF59 administered to healthy adults. Vaccine 2010;28(38):6367-6373

60 Ray R, Meyer K, Banerjee A, et al. Characterization of antibodies induced by vaccination with hepatitis $C$ virus envelope glycoproteins. J Infect Dis 2010;202(6):862-866

61 Law JL, Chen C, Wong J, et al. A hepatitis C virus (HCV) vaccine comprising envelope glycoproteins gpE1/gpE2 derived from a single isolate elicits broad cross-genotype neutralizing antibodies in humans. PLoS ONE 2013;8(3):e59776

62 Forns X, Emerson SU, Tobin GJ, Mushahwar IK, Purcell RH, Bukh J. DNA immunization of mice and macaques with plasmids encoding hepatitis $C$ virus envelope E2 protein expressed intracellularly and on the cell surface. Vaccine 1999;17(15-16):1992-2002

63 Garrone P, Fluckiger AC, Mangeot PE, et al. A prime-boost strategy using virus-like particles pseudotyped for HCV proteins triggers broadly neutralizing antibodies in macaques. Sci Transl Med 2011; 3(94):94ra71

64 Kong L, Giang E, Nieusma T, et al. Hepatitis C virus E2 envelope glycoprotein core structure. Science 2013;342(6162):1090-1094

65 Townsend AR, Rothbard J, Gotch FM, Bahadur G, Wraith D, McMichael AJ. The epitopes of influenza nucleoprotein recognized by cytotoxic $T$ lymphocytes can be defined with short synthetic peptides. Cell 1986;44(6):959-968

66 Blum JS, Wearsch PA, Cresswell P. Pathways of antigen processing. Annu Rev Immunol 2013;31:443-473

67 Ward S, Lauer G, Isba R, Walker B, Klenerman P. Cellular immune responses against hepatitis $C$ virus: the evidence base 2002 . Clin Exp Immunol 2002;128(2):195-203

68 Elmowalid GA, Qiao M, Jeong SH, et al. Immunization with hepatitis $C$ virus-like particles results in control of hepatitis $C$ virus infection in chimpanzees. Proc Natl Acad Sci U S A 2007; 104(20):8427-8432

69 Capone S, Meola A, Ercole BB, et al. A novel adenovirus type 6 (Ad6)-based hepatitis $C$ virus vector that overcomes preexisting anti-ad5 immunity and induces potent and broad cellular immune responses in rhesus macaques. J Virol 2006;80(4):1688-1699

70 Fattori E, Zampaglione I, Arcuri M, et al. Efficient immunization of rhesus macaques with an HCV candidate vaccine by heterologous priming-boosting with novel adenoviral vectors based on different serotypes. Gene Ther 2006;13(14):1088-1096

71 Jeong SH, Qiao M, Nascimbeni M, et al. Immunization with hepatitis $C$ virus-like particles induces humoral and cellular immune responses in nonhuman primates. J Virol 2004;78(13):6995-7003

72 Lang Kuhs KA, Ginsberg AA, Yan J, et al. Hepatitis C virus NS3/NS4A DNA vaccine induces multiepitope $T$ cell responses in rhesus macaques mimicking human immune responses. Mol Ther 2012;20(3):669-678

73 Polakos NK, Drane D, Cox J, et al. Characterization of hepatitis C virus core-specific immune responses primed in rhesus macaques by a nonclassical ISCOM vaccine. J Immunol 2001;166(5):3589-3598

74 Rollier C, Verschoor EJ, Paranhos-Baccala G, et al. Modulation of vaccine-induced immune responses to hepatitis $C$ virus in rhesus macaques by altering priming before adenovirus boosting. J Infect Dis 2005;192(5):920-929

75 Rollier C, Depla E, Drexhage JA, et al. Control of heterologous hepatitis $C$ virus infection in chimpanzees is associated with the quality of vaccine-induced peripheral T-helper immune response. J Virol 2004;78(1):187-196

76 Folgori A, Capone S, Ruggeri L, et al. A T-cell HCV vaccine eliciting effective immunity against heterologous virus challenge in chimpanzees. Nat Med 2006;12(2):190-197 
88 Will There Be a Vaccine to Prevent HCV Infection? Honegger et al.

77 Youn JW, Hu YW, Tricoche N, et al. Evidence for protection against chronic hepatitis $C$ virus infection in chimpanzees by immunization with replicating recombinant vaccinia virus. J Virol 2008; 82(21):10896-10905

78 Rollier CS, Paranhos-Baccala G, Verschoor EJ, et al. Vaccine-induced early control of hepatitis $C$ virus infection in chimpanzees fails to impact on hepatic PD-1 and chronicity. Hepatology 2007;45(3): 602-613

79 Park SH, Shin EC, Capone S, et al. Successful vaccination induces multifunctional memory T-cell precursors associated with early control of hepatitis C virus. Gastroenterology 2012;143(4): 1048-1060, e4

80 Barnes E, Folgori A, Capone S, et al. Novel adenovirus-based vaccines induce broad and sustained $\mathrm{T}$ cell responses to $\mathrm{HCV}$ in man. Sci Transl Med 2012;4(115):ra1
81 Cox AL, Thomas DL. Hepatitis C virus vaccines among people who inject drugs. Clin Infect Dis 2013;57(Suppl 2):S46-S50

82 Maher L, White B, Hellard M, et al. Candidate hepatitis C vaccine trials and people who inject drugs: challenges and opportunities. Vaccine 2010;28(45):7273-7278

83 Cox AL, Page K, Bruneau J, et al. Rare birds in North America: acute hepatitis C cohorts. Gastroenterology 2009;136(1):26-31

84 Altevogt BM, Pankevich DE, Shelton-Davenport MK, Kahn JP, eds; Committee on the Use of Chimpanzees in Biomedical and Behavioral Research; National Research Council. Chimpanzees in Biomedical and Behavioral Research: Assessing the Necessity. Washington DC: National Academy of Sciences; 2011

85 Billerbeck E, de Jong Y, Dorner M, de la Fuente C, Ploss A. Animal models for hepatitis C. Curr Top Microbiol Immunol 2013; 369:49-86 